\title{
Superconducting Tunnel Junction X-Ray Detectors for High Resolution Spectroscopy
}

S E Labov, M Fiank, J.B. le Grand, M A Lindeman, H Netel, L J Hiller, D. Chow, S. Friedıich, C A. Mears, G Caldara and A T Barfknecht

June 1, 1998

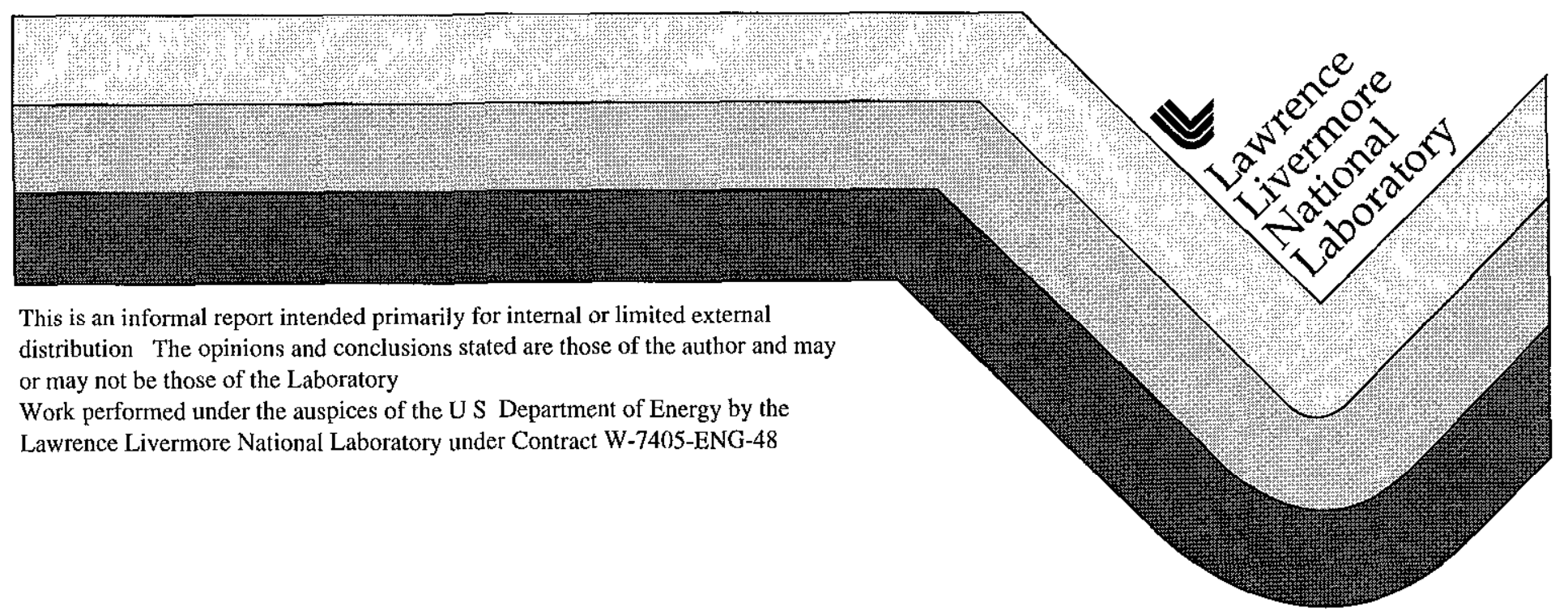




\section{DISCLAIMER}

This document was prepared as an account of work sponsored by an agency of the United States Government Neither the United States Government nor the University of California nor any of their employees, makes any warranty, express or implied, or assumes any legal liability or responsibility for the accuracy, completeness, or usefulness of any information, apparatus, product, or process disclosed, or represents that its use would not infringe privately owned rights Reference herein to any specific commercial product, process, or service by trade name, trademark, manufacturer, or otherwise, does not necessarily constitute or imply its endorsement, recommendation, or favoring by the United States Government or the University of California The views and opinions of authors expressed herein do not necessarily state or reflect those of the United States Government or the University of California, and shall not be used for advertising or product endorsement purposes

This report has been reproduced directly from the best available copy

Available to DOE and DOE contractors from the Office of Scientific and Technical Information

P O Box 62, Oak Ridge, TN 37831

Prices available from (423) 576-8401

Available to the public from the National Technical Information Service

US Department of Commerce 5285 Port Royal Rd, Springfield, VA 22161 


\title{
Superconducting Tunnel Junction X-ray Detectors for High Resolution Spectroscopy
}

\author{
Simon E Labov ${ }^{1}, M$ Frank $^{1}$, J B le Grand ${ }^{1}, \mathrm{M} \mathrm{A} \mathrm{Lindeman}^{1}$, H Netel $^{1}$, L J Hiller ${ }^{\mathrm{I}}, \mathrm{D}$ Chow ${ }^{1}$,

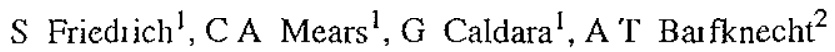 \\ ${ }^{1}$ Lawrence Livermore National Laboratory, P O Box 808, L-401, Livermore CA 94550, USA \\ ${ }^{2}$ Conductus, Inc , 969 West Maude Ave, Sunnyvale, CA 94086, USA
}

\section{Introduction}

We are developing low-temperature detectors for optical, ultraviolet, $X$-ray, and gamma-ray spectroscopy, and for biomolecular mass spectrometry We present here a some of our recent work in developing these detectors and some of the first results in applying these detectors to X-ray fluorescence analysis We have measured thin-film $\mathrm{Nb} / \mathrm{Al} / \mathrm{Al}_{2} \mathrm{O}_{3} / \mathrm{Al} / \mathrm{Nb}$ superconducting tunnel junction (STJ) X-ray detectors in the 02 to $1 \mathrm{keV}$ band with a range of different junction sizes and aluminum film thicknesses In one case, we have achieved the statistical limit to the energy resolution in this band We have measured the performance of these STJ detectors as a function of count rate and demonstrated a resolution of $13 \mathrm{eV} \mathrm{FWHM}$ at $277 \mathrm{eV}$ with an output count rate of 20,600 $\mathrm{cts} / \mathrm{s}$ Using $\mathrm{X}$ rays from SSRL to study compesite materials, we have demonstrated that we can resolve the $L$ lines of transition metals from the nearby $\mathrm{K}$ lines of light elements We describe the first use of a low-temperature X-ray detector to measure $\mathrm{X}$-ray fluorescence from the dilute metal component in a protein

\section{STJ detectors}

We have developed a $\mathrm{Nb} / \mathrm{Al} / \mathrm{Al}_{2} \mathrm{O}_{3} / \mathrm{Al} / \mathrm{Nb}$ superconducting tunnel junction (STJ) production process at Conductus Inc that allows us to fabricate detectors with very thin and very uniform $\mathrm{Al}_{2} \mathrm{O}_{3}$ tunnel barriers [1] Using these devices and a dc SQUID-array current amplifier, we measured a resolution of $29 \mathrm{eV}$ FWHM at $6 \mathrm{keV}[2,3]$ The SQUID amplifier also allowed us to obtain a resolution of $21 \mathrm{eV} \mathrm{FWHM}$ at $26 \mathrm{keV}$ with a detector $282 \times 282 \mu \mathrm{m}^{2}$ [4] Measurements between 1 and $8 \mathrm{keV}$ show a very linear response [5] These detectors, however, perform best at X-ray energies below $1 \mathrm{keV}$ At these energies the detectors are nearly $100 \%$ efficient, and we previously obtained a resolution of $125 \mathrm{eV} \mathrm{FWHM} \mathrm{at} 1 \mathrm{keV}$ [6] This device was measured with a standard FET-based current amplifier, and used a thin $50 \mathrm{~nm}$ aluminum "trapping" layer which allowed operation at temperatures up to $600 \mathrm{mK}$

In Fig 1 we show a schematic cross section of our STJ detectors The detectors consist of a $265 \mathrm{~nm}$ thick $\mathrm{Nb}$ base layer and a $165 \mathrm{~nm}$ thick $\mathrm{Nb}$ counter electrode separated by a thin $(-20 \AA) \mathrm{Al}_{2} \mathrm{O}_{3}$ tunnel barrier with $\mathrm{Al}$ "trapping" layers on each side of the barrier The thickness of the Al trapping layers range from 35 to 200 thick The detectors are diamond-shaped with sizes ranging from $20 \times 20 \mu \mathrm{m}^{2}$ to $200 \times 200 \mu \mathrm{m}^{2}$ These detectors were fabricated at Conductus, Inc, using a modified photolithographic $\mathrm{Nb}$ trilayer process [1] The $\mathrm{SiO}_{2}$ layer covering the devices was removed to allow low-energy $\mathrm{X}$-rays to reach the detector During operation a small magnetic field $(B \sim 10 \mathrm{mT})$ is applied parallel to the tunnel barrier in order to suppress the dc Josephson current in the device This suppression is necessary to allow stable operation of the device when biased near zero voltage

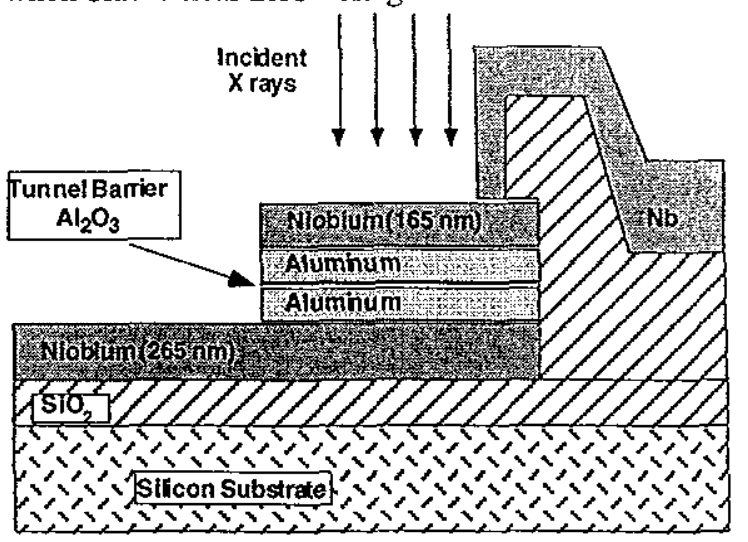

Fig 1 Cross section of STJ X-ray detector

During operation the detector is cooled to well below the critical temperature of the superconducting layers such that nearly all conduction electrons are bound into Cooper pairs and the number of thermal 
excitations is small For the 50 nm-thick Al trap devices, this is the case below $\sim 500 \mathrm{mK}$ The detectors with $200 \mathrm{~nm}$-thick $\mathrm{Al}$ traps operate best below $300 \mathrm{mK}$ The absorption of an X-ray photon in one of the superconducting electrodes breaks Cooper pairs creating quasiparticles, which can quantum-mechanically tunnel through the $\mathrm{Al}_{2} \mathrm{O}_{3}$ barrier When a small bias voltage is applied across this tunnel barrier the tunneling of the quasiparticles creates a measurable current signal The amplitude of the current pulse is proportional to the number of quasiparticles produced and thus to the energy of the absorbed X-ray photon

We refer to the Al layers as quasiparticle traps The process of "quasiparticle trapping" [7] relies on the fact that the Al layers have a lower superconducting energy gap than the $\mathrm{Nb}$ layers Therefore, when the quasiparticles diffuse to one of the Al layers they can relax energetically by emitting a phonon With a coriespondingly lower energy they cannot return into the $\mathrm{Nb}$ and thus become trapped in the $\mathrm{Al}$ This concentrates quasiparticles near the tunnel barrier increasing the tunnel rate and hence the signal The traps also reduces quasiparticles losses because the quasiparticle loss rate tends to be higher in $\mathrm{Nb}$ than in $\mathrm{Al}$

A schematic of the experimental setup used for the measurements discussed here is shown in Fig 2 The STJ detector was housed in a pumped liquid helium cryostat equipped with an adiabatic demagnetization refrigerator (ADR) unit with a base temperature of $\sim 50 \mathrm{mK}[8,9]$ During the experiments the temperature was not regulated and allowed to drift up freely

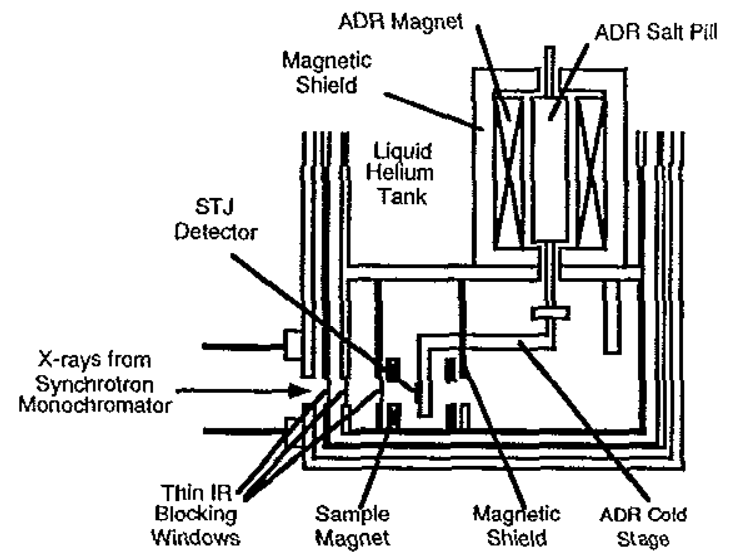

Fig 2 Schematic of the experimental setup

The X-ray experiments presented here were performed at the Stanford Synchrotron Radiation
Laboratory (SSRL) The cryostat was mounted onto an xyz stage and connected to the synchrotron bcam line with a flexible bellows By moving the cryostat we could align the detector with the synchrotron beam Moving the detector in and out of the center of the beam provided a convenient way of adjusting the coum rate Three thin windows were placed into the $77 \mathrm{~K}$ shield, the $2 \mathrm{~K}$ shield and the magnetic shield (also at $2 \mathrm{~K}$ ) in front of the detectors to limit the exposure of the detectors to infraied radiation emitted from the beam line at 300 $\mathrm{K}$ These windows consisted of $200 \AA$ aluminum and $1000 \AA$ of parylene on an $80 \%$ open Ni mesh

We used an FET-based preamplifier with fast ( 025 to $10 \mu \mathrm{s}$ ) negative feedback to measure the current signal from the STJ detector The rise time of the current pulses was limited by this amplifier to typically $\sim 05 \mu \mathrm{s}$, the decay time of the current pulses was given by the quasiparticle life time During most measurements we also injected pulses with similar shape from a pulse generator into the electronics to monitor the electronic noise The $\mathrm{X}$ ray induced current pulses and pulses from the pulse generator were further amplified and shaped either with an Ithaco 4302 filter amplifier with adjustable band pass or with a Canberra 2020 spectroscopy amplifier with a baseline restorer The shaped pulses were then fed into a pulse height analyzer without any further signal processing No pile-up rejection was used

\section{Theoretical Resolution Achieved with Soft $X$ rays}

As described below, we measured a series of 16 different detectors with four different sizes and four different $\mathrm{Al}$ trapping layer thickness of the 16 detectors measured for this study, the best overall resolution was obtained with the $50 \times 50 \mu \mathrm{m}^{2}$ detector with $200 \mathrm{~nm}$-thick $\mathrm{Al}$ trapping layers The spectra are very clean and free of artifacts, and the detector response is almost linear An example of a spectrum is shown in Fig 3 This spectrum shows three very narrow peaks The middle peak shows the response to $700 \mathrm{eV} \mathrm{X}$ rays, the other two peaks are due to the test pulser Below the $\mathrm{X}$-ray peak, some counts are seen which are due to white light scattered from the grating of the monochromator In spectra up to $650 \mathrm{eV}$ the shape of the X-ray peaks are very Gaussian For X-ray energies of $700-1000 \mathrm{eV}$, the central part of the lines are very Gaussian, but additional wings were present on both the low and the high energy sides of the lines 


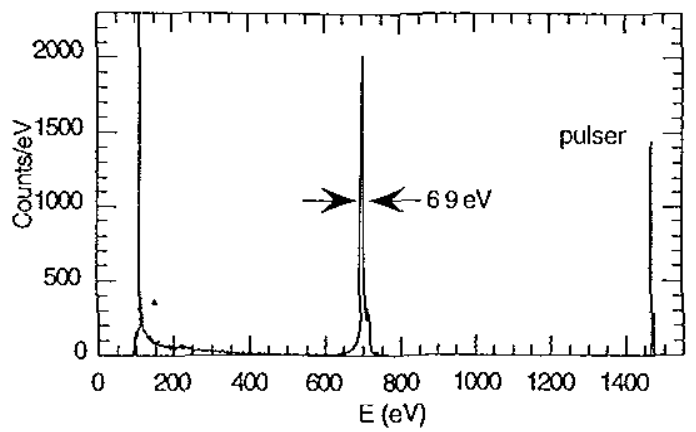

Fig $3 \mathrm{X}$-ray spectrum at $700 \mathrm{eV}$ obtained with 50 $\times 50 \mu \mathrm{m}^{2}$ STJ detector with 200 nm-thick Al trapping layers

In Fig 4 we show the resolution of this detector as a function of energy The solid squares in Fig 4 indicate the line widths obtained by measuring the width of the actual line at half the observed maximum height Neither fitting, nor continuum or tail subtractions were used The resolution ranges from $44 \mathrm{eV} \mathrm{FWHM}$ at $200 \mathrm{eV}$ to $116 \mathrm{eV}$ FWHM at 1 $\mathrm{keV}$ Some of the measured width at energies above $700 \mathrm{eV}$ is due to the energy width of the monochromator This contribution is indicated by the dotted line in Fig 4

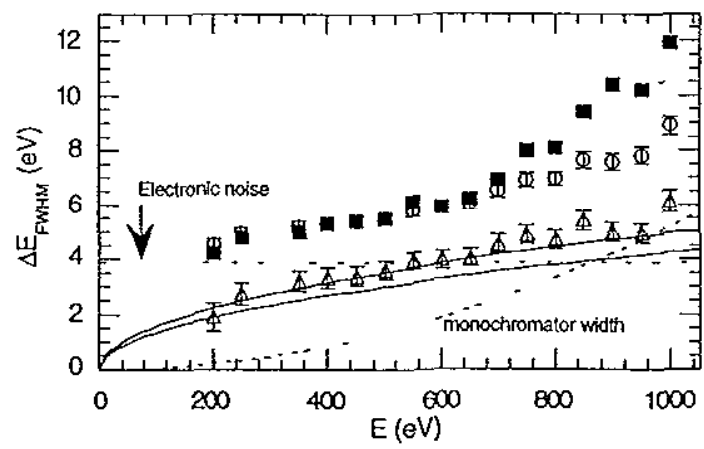

Fig 4 Resolution of $50 \times 50 \mu \mathrm{m}^{2}$ STJ detector with $200 \mathrm{~nm}$-thick Al trapping layers Squares show full width, open circles show width from a fit, open triangles show the intrinsic resolution Upper and lower solid lines show the theoretical resolution with and without quasiparticle multiplication respectively

To understand why the peaks have the observed shape and width, we fit the peaks to a Gaussian profile At energies below $600 \mathrm{eV}$, the Gaussian profile usually fits quite well, and the line widths from fitting agree with the line widths determined by directly measuring the FWHM At higher energies the additional structure in the wings must be taken into account This structure is typically asymmetric Below the peak there is a tail, while above the peak there is more often an extra small peak or bump The mechanisms which contribute to these features include $\mathrm{X}$ rays which are absorbed in the base electrode and Al layers [10], self-recombination [11], variations in the depth at which the $\mathrm{X}$ rays are absorbed, and residual $\mathrm{SiO}_{2}$ or other surface contaminates

To fit the central peak to a Gaussian profile we first subtract off any white light which scatters through the monochromator, and then we subtract off any non-Gaussian wing structure A Gaussian profile then fits quite well to the central peak We find that by varying how the wing structure is subtracted gives a variation in the width of about $4 \%$ The widths derived from fitting just the central part of the peak are indicated by circles in Fig 4

Now to determine the intrinsic resolution of the counter electrode, we subtract in quadrature the electronic noise and the intrinsic energy width of the Xray beam The electronic noise for this detector was $39 \mathrm{eV}$ FWHM, and is shown by the dashed line in Fig 4 For the monochrometer slit settings used, the energy width of the $\mathrm{X}$-ray beam increases quadratically with energy reaching $52 \mathrm{eV}$ at 1000 $\mathrm{eV}$, as shown by the dotted line in Fig 4 The intrinsic resolution, indicated by triangles in Fig 4, varies with the square root of the energy Fitting the width as a function of energy we find

$$
\Delta E_{F W H M}=\left(\begin{array}{lll}
0 & 170 \pm 0 & 014
\end{array}\right) \sqrt{E}
$$

with $\Delta E$ and $E$ in $\mathrm{eV}$

The theoretical resolution of a symmetric junction without quasiparticle multiplication is

$$
\Delta E_{F W H M}=235 \sqrt{\varepsilon\left(\overline{\left.F+F^{\prime}\right) E}\right.}
$$

where $\varepsilon$ is the average energy required to produce one quasiparticle, $F$ is the Fano factor describing the statistical distribution in the number of quasiparticles created The $F^{\prime}$ term, which was originally described by Mears et al [12] and later expanded by Goldie et al [13], accounts for the additional statistical fluctuations due to multiple tunneling of the quasiparticles back and forth through the tunnel barrier For $\mathrm{Nb}$ we assume $F=02$ and $\varepsilon=17 \Delta_{\mathrm{Nb}}[14,15]$ For symmetric junctions $F^{\prime}$ $=1+1 / n$ where $n$ is the average number of times each quasiparticle tunnels through the barrier For 
this detector $n \approx 13$ so the theoretical resolution without quasiparticle multiplication is

$$
\triangle E_{F W H M}=0134 \sqrt{E}
$$

which is plotted as a solid line in Fig 4

The measured result in Eq 1 is very close to the calculated resolution in $\mathrm{Eq} 3$ Moreover the remaining small difference can be explained by the fact that for the detectors with $200 \mathrm{~nm}$ of $\mathrm{Al}$, the measured gap in the $\mathrm{Al} \Delta \mathrm{Al}=034 \mathrm{meV}$ is less than a third of the $\mathrm{Nb}$ gap $\Delta_{\mathrm{Nb}}=15 \mathrm{meV}$ When a quasiparticle is trapped from the $\mathrm{Nb}$ into the $\mathrm{Al}$, a phonon as large as $116 \mathrm{meV}$ may be produced, which is energetic enough to break up another Cooper pair in the Al producing two more quasiparticles Since not every quasiparticle that gets trapped will multiply, statistical fluctuations in the number of quasiparticles produced during this multiplication will further broaden the resolution [16] We find this multiplication can increase the expected resolution up to

$$
\Delta E_{F W H M}=0157 \sqrt{E}
$$

This is shown by the solid line in Fig 4 which is within the error of the measured resolution $\mathrm{We}$ therefore conclude that the intrinsic resolution we measured for this detector is well described by the theory including the statistical fluctuations in the number of quasiparticles that are created, the fluctuations in the number of quasiparticles produced in multiplication and the fluctuations in the number of quasiparticles tunneling through the barrier

\section{Resolution at High Count Rates}

One of the advantages of superconductor insulator superconductor STJ X-ray detectors is their fast response The length of the current pulse we observe is determined by the quasiparticle lifetime in the device For the junctions with 50 nm-thick $\mathrm{Al}$ trapping layers, this was about $45 \mu$ s This fast response allows operation at much higher count rates than thermal microcalorimeters [17-20]

To explore the count rate capability of these detectors, we measured a detector $141 \times 141 \mu \mathrm{m}^{2}$ with $50 \mathrm{~nm}$ thick Al trapping layers We irradiated this detector with $277 \mathrm{eV} \mathrm{X}$ rays which corresponds to the energy of carbon $K$ The count rate was adjusted to the desired values by adjusting the cryostat position and moving the detector closer to the center of the synchrotron beam The Canberra 2020 spectroscopy amplifier was used including its automatic baseline restorer For count rates up to $10,000 \mathrm{cts} / \mathrm{s}$ optimal results were achieved with shaping times of $3-4 \mu \mathrm{s}$, above $10,000 \mathrm{cts} / \mathrm{s}$ with $15 \mu$ s No pile-up rejection was applied

At the low count rate of $375 \mathrm{cts} / \mathrm{s}$ the resolution was $59 \pm 01 \mathrm{eV}$ (FWHM), as shown in Fig 5 The electronic noise in this measurement was $45 \pm$ $01 \mathrm{eV}$ (FWHM)

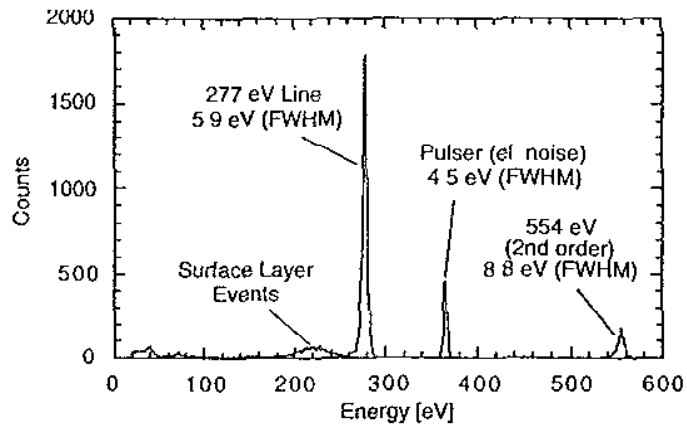

Fig $5 \quad \mathrm{X}$-ray spectrum at $277 \mathrm{eV}$ obtained with $141 \times 141 \mu^{2}$ STJ detector with $50 \mathrm{~nm}$-thick Al trapping layers Count rate was $375 \mathrm{cts} / \mathrm{s}$

In Fig 6 we show an $X$ ray spectrum measured at a count rate of $23,300 \mathrm{cts} / \mathrm{s}$ The count rate quoted here was the output count rate of the pulse height analyzer as determined from the total number of counts in the spectrum and the active time of the pulse height analyzer excluding the dead time, which was $13 \%$ in this measurement The $277 \mathrm{eV}$ line is resolved with a FWHM energy resolution of $130 \mathrm{eV} \pm 01 \mathrm{eV}$ The energy calibration was performed using the second-order X-ray line at $554 \mathrm{eV}$ resolved with $157 \pm 02 \mathrm{eV}$ (FWHM) The resolution was largely dominated by the electronic noise of $119 \pm 01 \mathrm{eV}$ as measured from the width of the pulser line The increase of this electronic noise compared to the electronic noise measured at low count rate was caused by baseline fluctuations resulting from the large count rate, pulse pile-up and

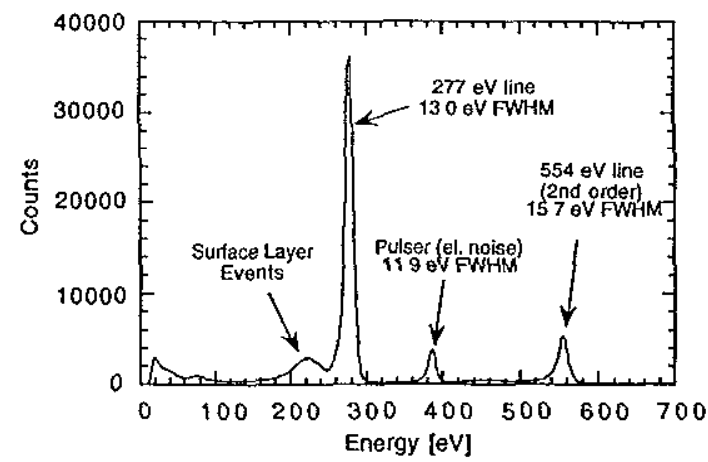

Fig 6 Same as Fig 5 but with count rate of $23,300 \mathrm{cts} / \mathrm{s}$ 
imperfect baseline restoration The contribution of the intrinsic energy width of the beam was negligible

In Fig 7 the measured FWHM energy resolution at $277 \mathrm{eV}$ incident $\mathrm{X}-\mathrm{r}$ ay energy (filled circles) and $554 \mathrm{eV}$ (2nd order X-rays, filled squares) is plotted together with the measured electronic noise (open circles) as function of the count rate Increasing the count rate from low rates of several $100 \mathrm{~Hz}$, the resolution at $277 \mathrm{eV}$ remained nearly constant at 6$8 \mathrm{eV}$ FWHM up to a several $1000 \mathrm{cts} / \mathrm{s}$

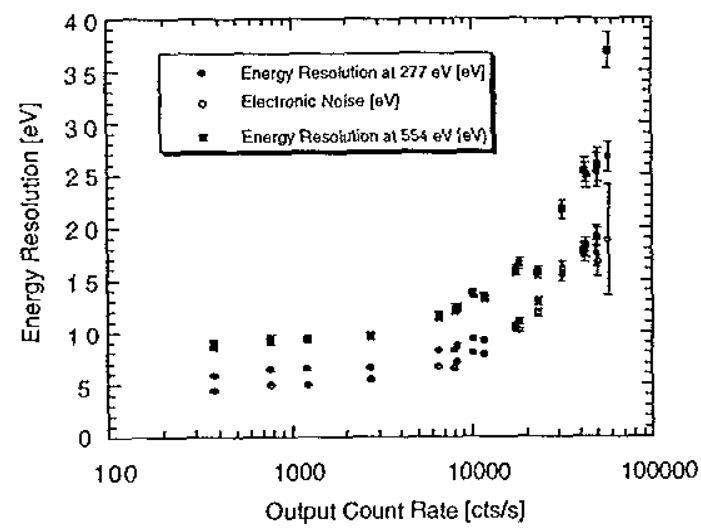

Fig 7 Total FWHM energy resolution at $277 \mathrm{eV}$

The resolution at $277 \mathrm{eV}$ remained below $10 \mathrm{eV}$ for count rates up to $\sim 10,000 \mathrm{cts} / \mathrm{s}$ and then degraded to $13 \mathrm{eV}$ at $23,000 \mathrm{cts} / \mathrm{s}$ and $20 \mathrm{cV}$ at $50,000 \mathrm{cts} / \mathrm{s}$ At $50,000 \mathrm{cts} / \mathrm{s}$ the PHA dead time approached $30 \%$ Above $50,000 \mathrm{cts} / \mathrm{s}$ the resolution seemed to degrade rapidly, presumably due to significant pulse pile up These results could probably be improved further by applying additional signal processing techniques such as pile-up rejection The high count rate measurements are discussed in more detail in Frank et al [21]

\section{STJ Performance as a Function of Al Thickness and Junction size}

To better understand the behavior of these devices, we fabricated a series of detectors with similar tunnel barrier characteristics but with different aluminum "trapping" layer thicknesses Previous measurements of this type have been performed with $6 \mathrm{keV} \mathrm{X}$ rays and similar detectors [22], and with detectors that have thinner Al layers [23] Here we describe measurements of detector response to soft X-rays from 02 to $1 \mathrm{keV}$, using detectors with both a range of aluminum trapping layer thickness, and a range of junction sizes
We measured detectors with four different $\mathrm{Al}$ trapping layer thicknesses $35 \mathrm{~mm}, 50 \mathrm{~nm}, 100 \mathrm{~nm}$ and $200 \mathrm{~nm}$ For each Al trap thickness, we measured detectors of four different sizes $20 \times 20 \mu \mathrm{m}^{2}, 50 \times$ $50 \mu \mathrm{m}^{2}, 70 \times 70 \mu \mathrm{m}^{2}$ and $141 \times 141 \mu \mathrm{m}^{2}$ making up for a total of 16 junctions We illuminated cach of these detectors with $\mathrm{X}$ rays sanging from $200 \mathrm{eV}$ to $1000 \mathrm{eV}$ in $50 \mathrm{eV}$ steps For all measurements similar conditions were used as much as possible The X-ray pulses were filtered using the Ithaco 4302 with a $1 \mathrm{MHz}$ low pass filter and a $315 \mathrm{kHz}$ high pass filter These settings appear to work reasonably well for all junctions even though the pulse length varied from 12 to $9 \mu \mathrm{s}$ We did not optimize the filter settings for each detector Instead, we chose a relatively large band pass of $315 \mathrm{kHz}$ to $1 \mathrm{MHz}$ for the pulse shaping in order to not distort the pulse shape too much While this way of pulse shaping is not optimal for achieving best energy resolution it allows us to compare the pulses from different STJs with decay times ranging from 12 to $9 \mu \mathrm{s}$

The bias voltage was roughly optimized for each junction, ranging from $02-03 \mathrm{mV}$ for the junctions with $200 \mathrm{~nm} \mathrm{Al} \mathrm{traps,} \mathrm{up} \mathrm{to} \mathrm{about} 05 \mathrm{mV}$ for the devices with $35 \mathrm{~nm} \mathrm{Al} \mathrm{traps} \mathrm{The} \mathrm{bias} \mathrm{cur-}$ rent varied strongly from junction to junction and not always proportional to the junction area, indicating either some residual trapped flux or variations in the quality of the junctions for these measurements the current amplifier had a feed back time of $1 \mu \mathrm{s}$ and the detector count rate was limited to $200 \mathrm{cts} / \mathrm{s}$ where the effect of count rate on energy resolution was negligible

Each peak arising from $X$ rays absorbed in the counter electrode was fit to a Gaussian When necessary to achieve a reasonable fit, the extra wing structures were subtracted before fitting a Gaussian to the central part of the line To find the line width intrinsic to each detector, the electronic noise and any contribution from the monochromator were subtracted in quadrature from the measured width

All junctions showed a fairly linear response The pulse height as a function of energy is well described by a second order polynomial with only a small quadratic correction.

$$
I_{c h}=A_{O}+A_{1} E+A_{2} E^{2}
$$

where $I_{c h}$ is the current pulse height in channels and $\mathrm{E}$ is the X-ray energy The offset $A_{0}$, comes from the analog to digital converter and is of no 
consequence As the nonlinearity is only a few percent, the detector response is mainly characterized by $A_{1}$ The $A_{2}$ term indicates the degree of nonlincarity in the detector

The width of the peaks were then analyzed as a function of energy For each junction we fit the width to a linear function of the energy and to a square root function of the energy We examined $\chi v^{2}$ for each fit to determine which type of dependence better characterized each detector If the resolution of a junction is proportional to the energy, then we infer that response varies with the location of the X-ray absorption If the resolution is proportional to the square root of the energy, then we infer a statistical process is involved

The response term $A_{1}$ is shown as a function of $\mathrm{Al}$ trap thickness in Fig 8

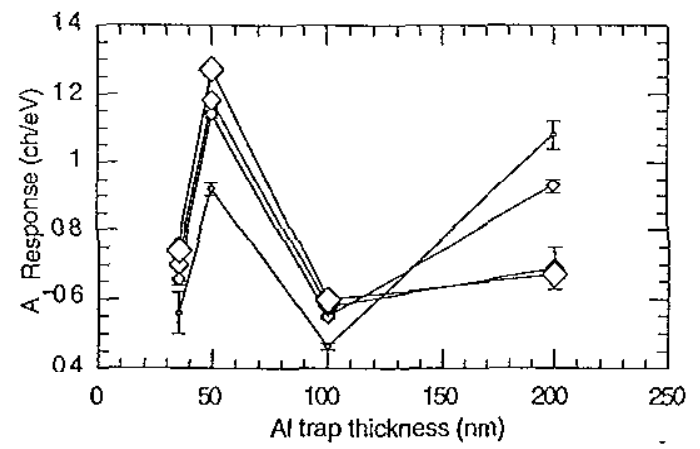

Fig 8 The STJ response $A_{1}$ as a function of Al trap thickness The size of the symbols relates to the size of the junctions

The highest response is from the detectors with $\mathrm{Al}$ trapping layers $50 \mathrm{~nm}$ thick For the detectors with $35 \mathrm{~nm}$-thick Al layers, the response is considerably smaller This is most likely due to the large energy gap in the thin Al layer and the slower trapping that results The junctions with $100 \mathrm{~nm}$-thick $\mathrm{Al}$ traps show less signal than the devices with $\mathrm{Al}$ $50 \mathrm{~nm}$ thick This is due to the lower tunneling rate The junctions with $\mathrm{Al}$ traps $200 \mathrm{~nm}$ thick show again a larger signal This is most probably caused by quasiparticle multiplication, as discussed in Section 2 above

In Fig 9 we plot the $A_{1}$ response term as a function of junction size There is a tendency of increasing signals with increasing size This is true except for the junctions with Al trapping layers $200 \mathrm{~nm}$ thick The effect is strongest for the $20 \times$
$20 \mu \mathrm{m}^{2}$ junctions For this size the self recombination of quasiparticles may be strong This is supported by the fact that these devices are also strongly nonlinear (see below) Also the proximity of edges and leads may provoke a decrease of the signal with decreasing size We do not yet understand the strong deviation in the behavior observed for the junctions with $200 \mathrm{~nm}$ thick Al trapping layers This may be due to magnetic flux trapped in the devices, although great care was taken to avoid trapped flux

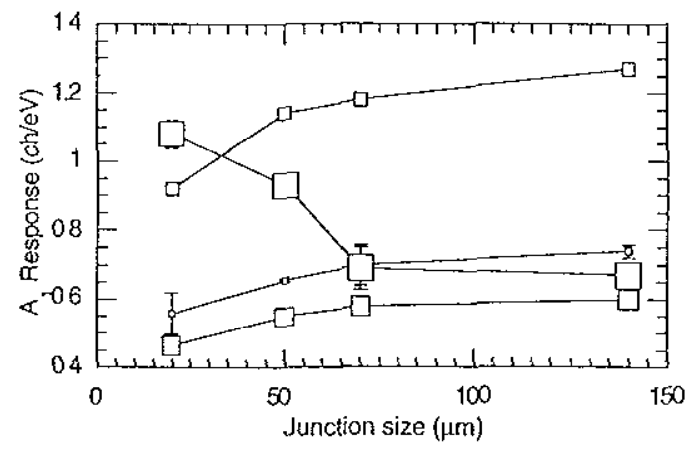

Fig 9 The STJ response $A_{1}$ as a function of junction size The size of the symbols relates to the thickness of the Al trapping layers

The nonlinearity coefficient $A_{2}$ is shown as a function of junction size in Fig 10

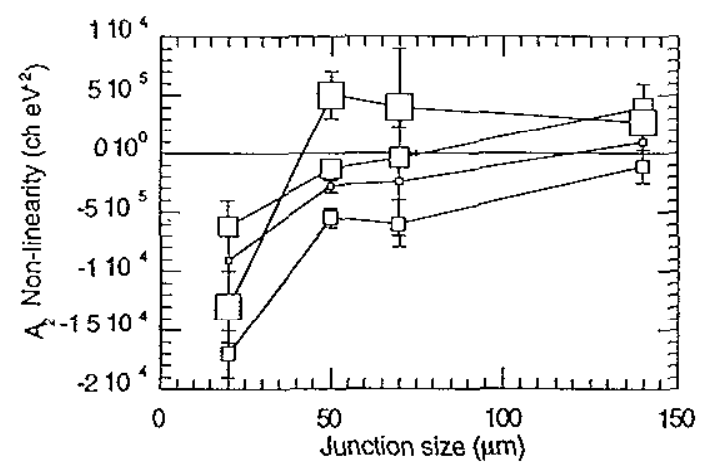

Fig 10 The nonlinearity coefficient $A_{2}$ as a function of junction size The size of the symbols relates to the thickness of the $\mathrm{Al}$ trapping layers

As evident in Fig 10, the response of the $20 \times 20$ $\mu \mathrm{m}^{2}$ junctions is significantly nonlinear This is due to self-recombination For most of the $50 \times$ $50 \mu \mathrm{m}^{2}$ junctions the signal is still slightly nonlinear For the larger junctions the response is linear, with $A_{2}$ close to zero 
For most of the detectors the intrinsic resolution was best fit to a linear function of energy For some of the better junctions with 50 or $200 \mathrm{~nm}$ thick Al layers, the square root dependence is a much better fit In Fig 11 we show the intrinsic resolution of each detector at $1 \mathrm{keV}$ The resolution of the $20 \times 20 \mu \mathrm{m}^{2}$ detectors is particularly bad because of the proximity of the lead and the edges The junctions with $35 \mathrm{~nm}$-thick AI trapping layers also show poor resolution This is probably because the thin trapping layess are not very efficient at trapping quasiparticles, which means the quasiparticles will spend more time in the Nb layers where we expect a higher quasiparticle loss rate The resolution of the junctions with 100 nm-thick Al traps is slightly worse than the junctions with 50 and 200 nm-thick Al traps This is probably because of the small signals these junctions produce A more detailed discussion of these measurements is in preparation [24]

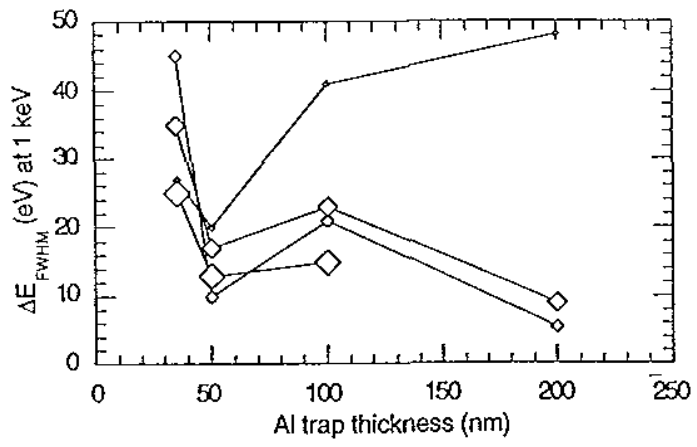

Fig 11 The intrinsic resolution of the STJ detectors as a function of Al trap thickness The size of the symbols relates to the size of the junctions

\section{X-ray fluorescence}

The performance of our STJ detectors below $1 \mathrm{keV}$ is very good, with energy resolution often below $10 \mathrm{eV}$, about ten times better than can be achieved with semiconductor ionization detectors Also, below $1 \mathrm{keV}$ the niobium counter electrode absorbs most of the incident X-ray photons To start taking advantage of the performance of these detectors, we have begun to use them in experiments requiring X-ray fluorescence analysis with high spectral resolution for soft $X$ rays For most of these experiments, we used a $141 \times 141 \mu \mathrm{m}^{2}$ detector with 50 nm-thick $\mathrm{Al}$ traps

In Fig 12 we show the X-ray fluorescence spectrum obtained with a sample consisting of boron nitride covered partially with titanium powder ex- cited by $500 \mathrm{eV} \mathrm{X}$ rays This sample was chosen to simulate $\mathrm{B}$ and TiN, which are important materials in semiconductor fabrication The $\mathrm{K}$ lines of $\mathrm{B}$, $\mathrm{N}$ and the $\mathrm{L}$ line of $\mathrm{Ti}$ are well separated in this spectrum Also present in this spectıum are $\mathrm{K}$ lines from $\mathrm{C}$ and $\mathrm{O}$, presumably from oxides and contamination in the sample, and an X-ray line at $500 \mathrm{eV}$ from scattered incident $X$ rays The FWHM energy resolution of the $\mathrm{X}$-ray lines ranges from $96 \mathrm{cV}$ for $\mathrm{C} \mathrm{K}$ to $131 \mathrm{eV}$ for $\mathrm{Ti} \mathrm{L}$ The electronic noise in this measurement was $67 \mathrm{eV}$ as indicated by the width of the pulser line For comparison, the resolution of $\mathrm{Si}(\mathrm{Li})$ detectors in this energy range is about a factor 10 worse and not sufficient to separate the $\mathrm{Ti} \mathrm{L}$ line from $\mathrm{N} \mathrm{K}$

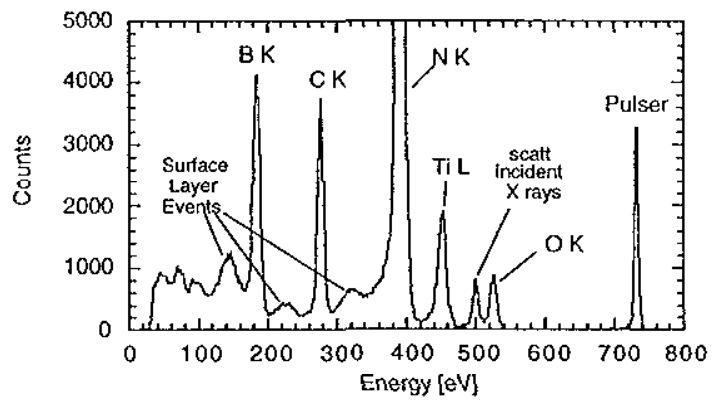

Fig 12 Fluorescence spectrum obtained with a BN sample coated with Ti powder

The $\mathrm{K}$ lines of $\mathrm{B}, \mathrm{C}$ and $\mathrm{N}$ in Fig 12 are each accompanied by a "hump" of events at the low-energy side of the line labeled "surface layer events" These humps probably originate from events caused by X-rays absorbed in a less responsive surface layer of our detector Such a surface layer may be composed of niobium oxide, residues of $\mathrm{SiO}_{2}$ from the detector fabrication process or other surface contamination These surface layers predominantly affects the lower energy $X$ rays We have found that detectors from some wafers do not show these artifacts The exact origin of these surface layer events and the other background seen at the low-energy end of the spectrum is still under study

In Fig 13 we show part of a fluorescence spectrum measured with a sample from a magnetic storage disk fabricated by IBM with $1000 \mathrm{eV}$ incident $\mathrm{X}$ rays The composition and thickness of the various metal layers in this sample is indicated on the right side of this figure Most of the L lines of the transition elements present in the sample (Cr, Co and $\mathrm{Ni}$ ) are resolved from each other and indications for 
some substructures of the lines are visible The resolution in the energy range shown was $10-15$ $\mathrm{eV}$ With these $L$ lines resolved one can, in principle, determine the composition and thickness of the various layers in the sample using the measured fluorescence intensities for the various elements and the fundamental parameter method [25]

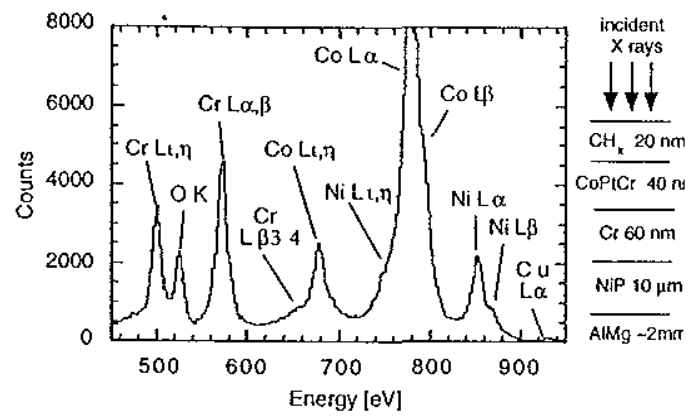

Fig 13 Fluorescence spectrum from a magnetic storage disk with $1 \mathrm{keV}$ incident $\mathrm{X}$-rays

In Fig 14 we show part of a fluorescence spectrum measured with a manganese oxide sample and an incident beam energy of $700 \mathrm{eV}$ The $\mathrm{Mn} \mathrm{L}$ lines are well resolved from the large $\mathrm{O} K$ line The $\mathrm{F} \mathrm{K}$ and $\mathrm{Fe} L$ lines are probably due to contamination of the sample The energy resolution of the $\mathrm{OK}$ line is $96 \mathrm{eV} \mathrm{FWHM}$ and the electronic noise was 46 $\mathrm{eV}$ With the measured resolution of $112 \mathrm{eV}$ at $640 \mathrm{eV}$ the line splitting between $\mathrm{Mn} \mathrm{L} \alpha$ and $\mathrm{L} \beta$ is visible the ability to measure $\mathrm{Mn}$ fluorescence in the presence of large amounts of $\mathrm{O}$ is interesting for various biological studies, e $g$ the study of photosystem II which is a protein containing Mn [26] These measurements are very difficult with conventional detectors because of significant line overlap between $\mathrm{O} \mathrm{K}$ and $\mathrm{Mn} \mathrm{L}$

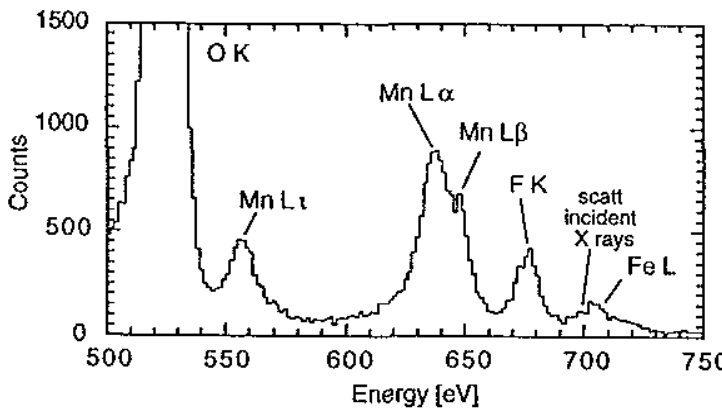

Fig 14 Fluorescence spectrum of MnO sample with incident beam energy of $700 \mathrm{eV}$
In Fig 15 we show the first $X$-ray fluorescence spectrum using an STJ detector to measure the $\mathrm{L}$ lines from a metalloprotein Here we used a $200 \times$ $200 \mu \mathrm{m}^{2}$ detector with $50 \mathrm{~nm}$-thick Al traps The sample was ferredoxin, a protein containing iron The incident energy was $780 \mathrm{eV}$ with an energy width of about $20 \mathrm{eV}$ The resolution of the $\mathrm{OK}$ line was $11 \mathrm{eV}$ FWHM

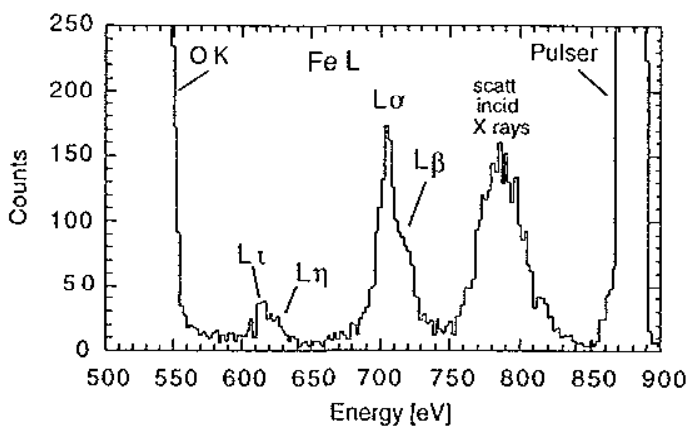

Fig 15 Fluorescence spectrum of ferredoxin sample with incident beam energy of $780 \mathrm{eV}$

\section{Acknowledgments}

We would like to thank Jan Batteux, Dennis Carr, Steve Cramer, Jeff Moore and Curtis Troxel for advice and expert technical support

This work was performed under the auspices of the U S Department of Energy by Lawrence Livermore National Laboratory under contract no W-7405ENG-48 Some experiments were performed at the Stanford Synchrotron Radiation Laboratory (SSRL) which is operated by the Department of Energy, Office of Basic Energy Sciences under contract number is DE-AC03-76SF00515 This work was also supported by NASA SBIR contract NAS5 . 32805, and NASA UV detector development grant NAG5 4137 JBIG acknowledges additional support from the Dutch Scientific Organization NWO

\section{References}

[1] A T Barfknecht, et al, IEEE Trans Mag MAG-27 (1991) 970

[2] M Frank, et al, NIM A 370 (1996) 41

[3] C A Mears, et al, NIM A370 (1996) 53

[4] C A Mears, et al, IEEE Trans Appl Supercon 7 (1997) 3415

[5] S E Labov, et al, IEEE Trans Appl Supercon 5 (1995) 3034

[6] L J Hiller, et al, SPIE Proc 2518 (1995) 249

[7] NE Booth, Appl Ph L 50 (1987) 293 
[8] P T Timbie, et al, Cryogenics 30 (1990) 271

[9] S Labov, et al, in Low Temperature Detectors for Neutrinos and Dark Matter IV, NE Booth and GL Salmon, Ed 1992, p 285

[10] C A Mears, et al, J X-ray Sci Tech (1997) in press

[11] J B 1 Grand, et al, Physica C 279 (1997) 85

[12] C A Mears, et al, Appl Ph L 63 (1993) 2961

[13] D J Goldie, et al, Appl Ph L 64 (1994) 3169

[14] M Kurakado, NIM 196 (1982) 275

[15] N Rando, et al, NIM 313 (1992) 173

[16] J B 1 Grand, et al, AppI Phys Lett (1997) submitted

[17] D McCammon, et al, NIM A326 (1993) 157

[18] E Silver, et al, X-Ray Spec 25 (1996) 115

[19] D A Wollman, et al, J Microscopy (1997) submitted

[20] S E Labov, et al, NIM A370) (1996) 65

[21] M Frank, et al, Rev Sci Inst 62 (1997) 25

[22] C A Mears, et al, LEEE Trans Appl Supeicon 5 (1995) 3069

[23] A Poelaert, et al, Proceedings SPIE 2808 (1996) 523

[24] J B 1 Grand, et al, (1997) in preparation

[25] M Mantler, Prog Crystal Growth and Charact 14 (1987)

[26] S P Cramer, et al, NIM A319 (1992) 285 


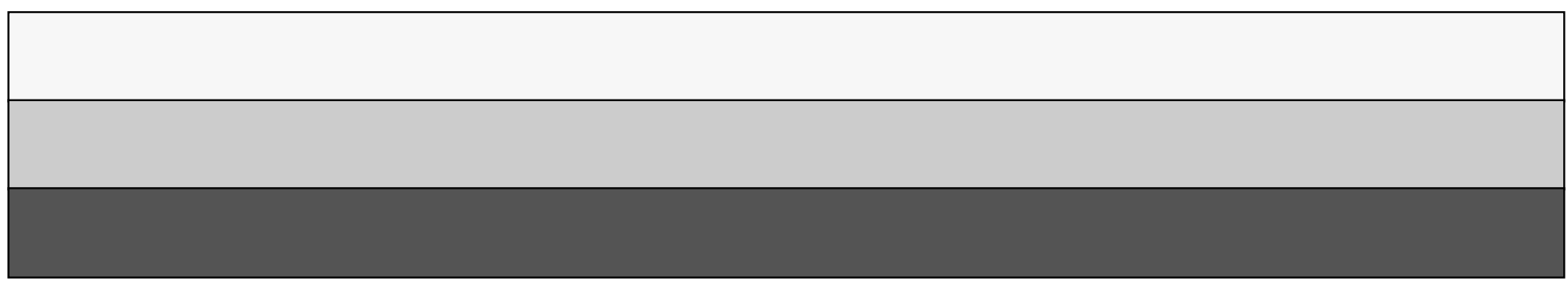

